of this size is likely to miss a clinically important 5\% difference in death rates between groups.

Another concern about this study is that the patient sample appeared to be of low rather than intermediate risk. This may have occurred because the protocol excluded patients with ST-segment depression in several leads and allowed emergency physicians to subjectively exclude patients with whom they were uncomfortable. In addition, although outcomes were statistically similar, the fact that there were 14 early MIs (6.6\%) in the non-CPU group versus 5 (2.4\%) in the CPU group may indicate that patients in the CPU group were healthier to begin with.

In this study and another by Gomez and associates, ${ }^{2}$ no cardiac events occurred among those CPU patients who were discharged after a negative stress test. Unfortunately both studies were underpowered and safety cannot be concluded.

Several studies cite cost efficacy as a major benefit of CPUs. Because these studies compare CPU costs only to costs for admitted patients, not to similar patients who were discharged from the ED with lesser work-ups, the true cost advantage of CPUs remains uncertain. Further, cost analysis may be inappropriate until the clinical safety of CPUs is better proven.

Published literature on CPUs suggests that discharging low- and intermediate-risk patients from the ED after a stable observation period and negative provocative test is a safe practice; however, before this can be recognized as the standard of care, a larger multicentre study or sufficiently powered systematic review should be undertaken.

\section{References}

1. Braunwald E, Jones RH, Mark DB, Brown J, Brown L, Cheitlin $\mathrm{MD}$, et al. Diagnosing and managing unstable angina. Quick Reference Guide for Clinicians. No 10. Rockville (MD): National Heart, Lung, and Blood Institute; 1994. AHCPR publ. no. 94-0602.

2. Gomez MA, Anderson JL, Karagounis LA, Muhlstein JB, Mooers FB. An emergency department-based protocol for rapidly ruling out myocardial ischemia reduces hospital time and expense: results of a randomized study (ROMIO). J Am Coll Cardiol 1996;28:25-33.

\section{Editor's note:}

This study suggests that some patients with "rule-out" unstable angina can be safely discharged after an abbreviated CPU work-up. Unfortunately, because of the nature of the enrolment criteria, it's unclear which patients these are. The study also shows that if you normally admit low-risk patients to a CCU (unlikely in Canada), you can reduce costs by not doing so. The study doesn't address what will happen if we widely introduce CPUs and, by protocol, subject patients (who previously would have been discharged from the ED) to intensive diagnostic algorithms. It is likely that this could lead to MORE investigations on more patients ("build it and they will come"), generating more costs, without clear evidence of improved patient outcomes.[G.I.]

\title{
Our evidence-based future
}

\section{Section Editors' notes:}

Evidence-based medicine (EBM) has a growing role in health care. Britain's National Health Service has mandated the development of a wide variety of evidence-based care plans, which they intend to introduce nationwide on a compulsory basis. Our own federal Health Minister is already discussing the importance of valid health outcomes research, so whether we believe in the concept or not, it is likely that the EBM wave will wash over Canada in the near future. To ensure that EBM is not abused, it is incumbent upon all of us to understand the process, its strengths, and its limitations.

We hope that many of you will submit CATs and evidence-based reviews for us to evaluate and choose from. If we receive more quality submissions than we can publish, we will arrange publication on the CAEP Web site. Please send us your reviews, your opinions, and any other suggestions you might have for innovations or additions to this section of CJEM. [D.R., M.B.] 